\title{
Fe-induced layer exchange of multilayer graphene for rechargeable battery anodes
}

\author{
Yoshiki Nakajima ${ }^{1, a)}$, Hiromasa Murata ${ }^{1, a)}$, Yuya Kado ${ }^{2, b)}$, Ryo Matsumura ${ }^{3}$, Naoki \\ Fukata $^{3}$, Takashi Suemasu ${ }^{1}$, and Kaoru Toko ${ }^{1, b)}$ \\ ${ }^{1}$ Institute of Applied Physics, University of Tsukuba, 1-1-1 Tennodai, Tsukuba, Ibaraki 305-8573, Japan \\ ${ }^{2}$ Research Institute of Energy Frontier, AIST, 16-1 Onogawa, Tsukuba 305-8569, Japan \\ ${ }^{3}$ National Institute for Materials Science, Namiki, Tsukuba 305-0044, Japan
}

Multilayer graphene (MLG) is a promising material for anodes of next-generation thin-film rechargeable batteries. The inverted layer exchange using an Fe catalyst allowed for the low-temperature $\left(600{ }^{\circ} \mathrm{C}\right)$ self-organization of the anode electrode structure, that is, an active material (MLG) on a current collector (Fe). A coin-type cell, fabricated from the MLG electrode and pure Li metal foil, showed distinct peaks, indicating Li intercalation into the MLG in a cyclic voltammogram. After 100 charge/discharge cycles, the capacity was 3.3 $\mu \mathrm{Ah} \mathrm{cm}^{-2}$ and coulomb efficiency was $98 \%$. The low-temperature synthesis of a MLG anode structure and its operation were demonstrated.

a) These authors contributed equally to this work.

b) Authors to whom correspondence should be addressed:

y.kado@aist.go.jp and toko@bk.tsukuba.ac.jp 
All-solid-state batteries are ideal power sources for many applications requiring high energy and power densities, good capacity retention for thousands of discharge/charge cycles, and high safety. ${ }^{1,2}$ Graphite, which has been used as an anode material in conventional lithium ion batteries (LIBs), exhibits good electrochemical performance even for solid electrolytes. ${ }^{3}$ The development of graphite anode synthesis technology will enable the fabrication of small and thin all-solid-state batteries suitable for mobile devices or sensors. ${ }^{4}$

Graphene, equivalent to one layer of graphite, has a very high specific capacity owing to its high surface area. ${ }^{5,6}$ However, using one graphene layer as an anode material is not practical from the viewpoint of capacity per area. Therefore, multilayer graphene (MLG) with a sufficient thickness is required. In addition, considering the heat resistance temperature of the substrate and the peripheral devices, the process temperature of MLG synthesis must be low. Therefore, a low-temperature synthesis technique for MLG on arbitrary substrates will bring the possibility of fabricating all-solid-state batteries anywhere. ${ }^{5}$

MLG has been produced on arbitrary substrates using transfer techniques, ${ }^{7}$ vapor deposition, ${ }^{8-14}$ and metal-induced solid-phase crystallization. ${ }^{15-29}$ However, it was difficult to synthesize a uniform and thick MLG at low temperature. We overcame these problems by developing metal-induced layer exchange (MILE) of amorphous C (a-C) ${ }^{30-33}$ In MILE, an a-C layer crystallizes through layer exchange between the a-C and the metal catalyst layer. MILE allows the reduction of the crystallization temperature of a-C and the formation of thick MLG on arbitrary substrates because the metal layer serves as both a catalyst and a "mold", i.e., the shape of the MLG after layer exchange becomes the initial shape of the metal layer. ${ }^{33} \mathrm{We}$ achieved layer exchange between $\mathrm{C}$ and the eight kinds of transition metals (Co, $\mathrm{Ni}, \mathrm{Cr}, \mathrm{Mn}$, $\mathrm{Fe}, \mathrm{Ru}, \mathrm{Ir}$, and $\mathrm{Pt}){ }^{32}$ Among the metals for MILE of $\mathrm{C}$, Fe is attractive because it is easily available. In this study, we investigate Fe-induced layer exchange of a-C in detail and demonstrate the LIB anode operation of the MLG. 
The sample preparation procedure of Fe-induced layer exchange is shown in Fig. 1(a). Fe and a-C thin films (50 nm thick each) were sequentially prepared on quartz glass $\left(\mathrm{SiO}_{2}\right)$ using radio-frequency $(\mathrm{RF})$ magnetron sputtering (base pressure: $3.0 \times 10^{-4} \mathrm{~Pa}$ ) with Ar plasma. The substrate temperature was $200{ }^{\circ} \mathrm{C}$ for both $\mathrm{Fe}$ and a-C. The RF power was set to $50 \mathrm{~W}$ for $\mathrm{Fe}$ and $100 \mathrm{~W}$ for a-C. The samples were annealed at $600-1000^{\circ} \mathrm{C}$ for $1 \mathrm{~h}$ in an $\mathrm{Ar}$ ambient pressure to form MLG on the substrate by layer exchange. The way for determining layer exchange is as follows: (i) The color of the front and back sides of the sample clearly changes after annealing. (ii) The MLG layer survives on the substrate after Fe removal using a diluted $\mathrm{HNO}_{3}$ solution (1.0\% $\left.\mathrm{HNO}_{3}\right)$.

Figure 1(b) shows that the color of the front and back sides of the sample clearly changes after annealing: the back side is the metallic color of Fe before annealing, but it becomes black from the carbon after annealing. Raman spectroscopy was performed using a JASCO NRS5100, wherein the laser wavelength was $532 \mathrm{~nm}$ and spot size was $5 \mu \mathrm{m}$. Figure 1(c) shows that the front and back sides of the samples have Raman peaks at approximately 1350, 1580, and $2700 \mathrm{~cm}^{-1}$, corresponding to the D, G, and G' peaks in the graphitic structure, respectively. ${ }^{34}$ We note that the peaks around 1100 and $1200 \mathrm{~cm}^{-1}$ are derived from the $\mathrm{SiO}_{2}$ substrate. The strong $G$ and $G$ ' peaks from the back side indicate that MLG is formed on the back side via layer exchange at all temperatures. The relatively large D peak from the front side is derived from the MLG "islands", which are a typical feature in MILE. ${ }^{31}$ To clarify the feature of Feinduced layer exchange, the crystal quality of the MLG was evaluated and compared with the MLG formed by Ni-induced layer exchange. From the Raman spectra, we evaluated the G-peak to D-peak intensity ratio, which indicates the crystal quality (the amount of the defects such as grain boundaries and point defects) of MLG. ${ }^{34}$ Figure $1(\mathrm{~d})$ shows that the G/D intensity ratio of the Fe samples is higher than that of the Ni samples at all annealing temperatures, indicating that the Fe provides the MLG, whose crystallinity is higher than the MLG formed using Ni. 
Generally, in layer exchange, the lower growth temperature provides the larger grain size and fewer grain boundaries, while it provides the more defects other than grain boundaries. ${ }^{35,36}$ For the Ni samples, the G/D intensity ratio is constant with the growth temperature, reflecting the balance between grain boundaries and point defects. ${ }^{30}$ Conversely, for the Fe samples, the higher growth temperature provides the larger G/D intensity ratio. Therefore, the temperature dependence of the G/D intensity ratio is different between the Ni samples and the Fe samples. Although the basic mechanism of layer exchange is considered to be the same, the growth process will be a little different. One possible reason is the drastic increase of the carbon solubility of Fe with the phase transition from $\alpha$-Fe to $\gamma$-Fe at $727^{\circ} \mathrm{C}$. Although the detailed mechanism is still unclear, the carbon solubility may affect the crystallinity of the resulting MLG.

The detailed cross-sectional structure of the sample annealed at $1000{ }^{\circ} \mathrm{C}$ was investigated using an analytical transmission electron microscope (TEM, FEI Tecnai Osiris). The crosssectional TEM samples were prepared using the conventional focused ion beam (FIB) method. The bright-field TEM image in Fig. 2(a) shows that the Fe layer is stacked on the MLG layer. This indicates that the MLG layer was directly formed on the $\mathrm{SiO}_{2}$ substrate by layer exchange between the a-C and Fe layers. The high-resolution TEM image in Fig. 2(b) clearly shows that the MLG layer is completely $\{002\}$-oriented. In contrast, for the Ni sample, the MLG layer was rippled and contained randomly oriented nanocrystalline $\mathrm{C}$ in the spaces between the rippled MLG and the Ni layer or substrate. ${ }^{30}$ This result properly accounts for the high crystallinity of MLG derived in the Raman spectra from the G/D ratio (Fig. 1(d)). The selected-area electron diffraction (SAED) pattern in Fig. 2(c) is spotty, indicating the high crystal quality of the MLG layer. The SAED pattern also reveals that C $\{002\}$ planes in the MLG are oriented almost parallel to the substrate. Although it was difficult to directly observe grain boundaries in the MLG layer in the TEM images, the SAED analyses indicated that the grain size was 
approximately a few hundred nm. In some parts, C islands are stacked on the bottom MLG layer, as representatively shown in Fig. 2(d). The island layers are responsible for the Raman peaks corresponding to MLG at the front side of the samples (Fig. 1(c)). After removing the Fe layers, the MLG layers survived on the substrates for all the samples, as representatively shown in Fig. 2(e). Figure 2(f) shows that the Fe concentration in the MLG layer is below the detection limit of an energy dispersive X-ray (EDX) analysis ( $<1 \%)$.

For the actual LIB structure, a current collector is required under the active anode material. Therefore, we investigated the self-organization of the current collector using “inverted” layer exchange. ${ }^{37,38}$ Here, we used a Mo foil as a substrate for anode characterization using a coin cell. The schematic of the inverted layer exchange is shown in Fig. 3(a). a-C and Fe thin films (each $200 \mathrm{~nm}$ thick) were sequentially prepared on Mo foil (50 $\mu \mathrm{m}$ thick). The deposition method is the same as the normal layer exchange. The samples were then annealed at $600{ }^{\circ} \mathrm{C}$ for $1 \mathrm{~h}$. Figure 3(b) shows that the front side of the sample has Raman peaks corresponding to the MLG after annealing. The cross-sectional structure of the sample was investigated using an FIB scanning electron microscope (SEM, FEI Helios NanoLab 600i), equipped with EDX. Because the EDX measurement is performed on a tilted sample, the signals of the materials behind are also detected. The FIB-SEM image in Fig. 3(c) shows the clear contrast of the stacked layer structure. Figure 3(d)-3(f) suggests that the C layer is stacked on the Fe layer. These results indicate that the MLG-anode on Fe-electrode structure was self-organized by the inverted layer exchange.

The sample was punched through a 10-mm-diameter disk, then used as electrodes after being dried $12 \mathrm{~h}$ in vacuum at $120^{\circ} \mathrm{C}$. Coin-type cells were fabricated from the MLG on the Fe (each $100 \mathrm{~nm}$ thick) electrode, pure Li metal foil, and separator (Celgard 2400), immersed in an electrolyte. The electrolyte was $1 \mathrm{~mol} \mathrm{~L}^{-1}$ lithium hexafluorophosphate $\left(\mathrm{LiPF}_{6}\right)$ in ethylene carbonate (EC)/diethyl carbonate (DEC) (1:1 in volume). A resulting coin cell is shown in the 
insertion in Fig. 3(a). The electrochemical characteristics of the coin cells were investigated using a multichannel galvanostat-potentiostat (Bio-Logic VMP). As shown in Fig. 4(a), the cyclic voltammogram (CV) of MLG shows two distinct peaks at 0.06 and $0.09 \mathrm{~V}$, in contrast to that of a bare Mo foil. These peaks correspond to the Li insertion into the MLG. ${ }^{39}$ Meanwhile, some parts of the CV curves of the MLG and bare Mo overlay closely, indicating that electric double-layer capacitance cannot be ignored. Figure 4(b) shows that the MLG exhibits charge/discharge operation. The first discharge capacity is anomalously high owing to a faradaic reduction of the electrolyte to form the solid electrolyte interface (SEI) at $0.8 \mathrm{~V} .^{40} \mathrm{In}$ the charge/discharge curves, the capacity gradually increased as the MLG was negatively charged, which is explained by the capacitive contribution and Li insertion in the MLG being more disordered than graphite, ${ }^{41}$ as indicated by the Raman study (Fig. 3(b)). ${ }^{42}$ The rapid increase in capacity was observed at lower voltages than $0.1 \mathrm{~V}$. This is attributable to $\mathrm{Li}$ intercalation in the local short-range ordered graphene layers, corresponding to the reduction/oxidation peak in the cyclic voltammogram (Fig. 4(a)). Figure 4(c) shows that the discharge capacity is $3.3 \mu \mathrm{Ah} \mathrm{cm}^{-2}$ after 100 cycles, which is $64 \%$ of the initial discharge capacity (5.1 $\mu \mathrm{Ah} \mathrm{cm}^{-2}$ ). Although the initial coulomb efficiency is $42 \%$, reflecting the irreversible capacity, it reaches approximately 98\% after 100 cycles. In general, in case of graphite which is a typical carbon anode of LIBs, through charge and discharge cycling, Li cointercalation with solvent molecules causes exfoliation of the graphene layers, resulting in the degradation of graphite anodes. Although a similar behavior is expected for MLG cases if we repeated the larger number of cycles, the change of the carbon structure will not be significant after 100 cycles. Further investigations on the durability are required in future work. Figure 4(d) shows the rate performance of the MLG, expressing the capacity at $66.6 \mu \mathrm{A} \mathrm{cm}^{-2}$ was $42 \%$ of the capacity at $0.7 \mu \mathrm{A} \mathrm{cm}^{-2}$. This is the superior performance to a commercial graphite, which could be related to the capacitive contribution and reduced diffusion distance of Li ion in the 
local short-range ordered structures. ${ }^{43-45}$ Although there is room for improvement in the capacity and the initial coulombic efficiency rate characteristics, we have demonstrated the anode operation of MLG formed at low temperature $\left(600^{\circ} \mathrm{C}\right)$.

In conclusion, we achieved MLG at low temperature using the Fe-induced layer exchange of a-C. The inverted layer exchange allowed for the self-organization of the MLG on an Feelectrode structure, exhibiting the LIB anode operation. The achievement pioneers the application of low-temperature MLG to promising anodes for next-generation thin-film rechargeable batteries.

This work was financially supported by the JSPS KAKENHI (No. 18K18844), Project for University-Industry Cooperation Strengthening in Tsukuba, and ATI Research Grants (RG2906). Some experiments were performed at the International Center for Young Scientists at NIMS and the Nanotechnology Platform at the University of Tsukuba. 


\section{REFERENCES}

1 J.F.M. Oudenhoven, L. Baggetto, and P.H.L. Notten, Adv. Energy Mater. 1, 10 (2011).

$2 \quad$ K. Takada, Acta Mater. 61, 759 (2013).

3 K. Takada, N. Ohta, L. Zhang, K. Fukuda, I. Sakaguchi, R. Ma, M. Osada, and T. Sasaki, Solid State Ionics 179, 1333 (2008).

$4 \quad$ N. J. Dudney, Mater. Sci. Eng. B 116, 245 (2005)

5 G. Radhakrishnan, J.D. Cardema, P.M. Adams, H.I. Kim, and B. Foran, J. Electrochem. Soc. 159, A752 (2012)

6 D. Wei, S. Haque, P. Andrew, J. Kivioja, T. Ryhänen, A. Pesquera, A. Centeno, B. Alonso, A. Chuvilin, and A. Zurutuza, J. Mater. Chem. A 1, 3177 (2013).

$7 \quad$ K.S. Novoselov, A.K. Geim, S. V. Morozov, D. Jiang, Y. Zhang, S. V. Dubonos, I. V. Grigorieva, and A.A. Firsov, Science 306, 666 (2004).

8 C.-Y. Su, A.-Y. Lu, C.-Y. Wu, Y.-T. Li, K.-K. Liu, W. Zhang, S.-Y. Lin, Z.-Y. Juang, Y.-L. Zhong, F.-R. Chen, and L.-J. Li, Nano Lett. 11, 3612 (2011).

$9 \quad$ T. Kato and R. Hatakeyama, ACS Nano 6, 8508 (2012).

10 W.-C. Yen, Y.-Z. Chen, C.-H. Yeh, J.-H. He, P.-W. Chiu, and Y.-L. Chueh, Sci. Rep. 4, 4739 (2014).

11 K. Murakami, S. Tanaka, A. Hirukawa, T. Hiyama, T. Kuwajima, E. Kano, M. Takeguchi, and J. Fujita, Appl. Phys. Lett. 106, 093112 (2015).

12 S. Chugh, R. Mehta, N. Lu, F.D. Dios, M.J. Kim, and Z. Chen, Carbon N. Y. 93, 393 (2015).

13 K. Ueno, H. Ichikawa, and T. Uchida, Jpn. J. Appl. Phys. 55, 04EC13 (2016).

14 Y. Nakajima, H. Murata, N. Saitoh, N. Yoshizawa, T. Suemasu, and K. Toko, ACS Omega 4, 6677 (2019).

15 Z. Peng, Z. Yan, Z. Sun, and J.M. Tour, ACS Nano 5, 8241 (2011).

16 J. Kwak, J.H. Chu, J.-K. Choi, S.-D. Park, H. Go, S.Y. Kim, K. Park, S.-D. Kim, Y.-W. Kim, E. Yoon, S. Kodambaka, and S.-Y. Kwon, Nat. Commun. 3, 645 (2012).

17 K. Banno, M. Mizuno, K. Fujita, T. Kubo, M. Miyoshi, T. Egawa, and T. Soga, Appl. Phys. Lett. 103, 082112 (2013).

18 W. Xiong, Y.S. Zhou, W.J. Hou, T. Guillemet, J.F. Silvain, Y. Gao, M. Lahaye, E. Lebraud, S. Xu, X.W. Wang, D.A. Cullen, K.L. More, L. Jiang, and Y.F. Lu, RSC Adv. 5, 99037 (2015).

19 D. Berman, S.A. Deshmukh, B. Narayanan, S.K.R.S.R.S. Sankaranarayanan, Z. Yan, A.A. Balandin, A. Zinovev, D. Rosenmann, and A. V. Sumant, Nat. Commun. 7, 12099 (2016). 
Vishwakarma, M.S. Rosmi, K. Takahashi, Y. Wakamatsu, Y. Yaakob, M.I. Araby, G. Kalita, M. Kitazawa, and M. Tanemura, Sci. Rep. 7, 43756 (2017).

S.J. Byun, H. Lim, G.Y. Shin, T.H. Han, S.H. Oh, J.H. Ahn, H.C. Choi, and T.W. Lee, J. Phys. Chem. Lett. 2, 493 (2011).

22 K. Gumi, Y. Ohno, K. Maehashi, K. Inoue, and K. Matsumoto, Jpn. J. Appl. Phys. 51, 06FD12 (2012). Nano Lett. 13, 4624 (2013). 53, 04EB05 (2014). Zhong, X.H. Sun, and S.T. Lee, ACS Nano 9, 594 (2015). 41664 (2018). (2014).

M. Tamaoki, H. Imaeda, S. Kishimoto, and T. Mizutani, Appl. Phys. Lett. 103, 183114 (2013).

H. Tanaka, S. Obata, and K. Saiki, Carbon N. Y. 59, 472 (2013).

M. Sato, M. Takahashi, H. Nakano, Y. Takakuwa, M. Nihei, S. Sato, and N. Yokoyama, Jpn. J. Appl. Phys.

M. Kosaka, S. Takano, K. Hasegawa, and S. Noda, Carbon N. Y. 82, 254 (2015).

Q.Q. Zhuo, Q. Wang, Y.P. Zhang, D. Zhang, Q.L. Li, C.H. Gao, Y.Q. Sun, L. Ding, Q.J. Sun, S.D. Wang, J.

J. Yamada, Y. Ueda, T. Maruyama, and S. Naritsuka, Jpn. J. Appl. Phys. 55, 100302 (2016).

H. Murata, K. Toko, N. Saitoh, N. Yoshizawa, and T. Suemasu, Appl. Phys. Lett. 110, 033108 (2017).

H. Murata, N. Saitoh, N. Yoshizawa, T. Suemasu, and K. Toko, Appl. Phys. Lett. 111, 243104 (2017).

Y. Nakajima, H. Murata, N. Saitoh, N. Yoshizawa, T. Suemasu, and K. Toko, ACS Appl. Mater. Interfaces 10, H. Murata, Y. Nakajima, N. Saitoh, N. Yoshizawa, T. Suemasu, and K. Toko, Sci. Rep. 9, 4068 (2019).

L.M. Malard, M.A. Pimenta, G. Dresselhaus, and M.S. Dresselhaus, Phys. Rep. 473, 51 (2009).

K. Toko, M. Kurosawa, N. Saitoh, N. Yoshizawa, N. Usami, M. Miyao, and T. Suemasu, Appl. Phys. Lett. 101, 072106 (2012).

K. Toko, R. Numata, N. Oya, N. Fukata, N. Usami, and T. Suemasu, Appl. Phys. Lett. 104, 022106

K. Toko, R. Numata, N. Saitoh, N. Yoshizawa, N. Usami, and T. Suemasu, J. Appl. Phys. 115, 094301 (2014).

K. Toko, K. Nakazawa, N. Saitoh, N. Yoshizawa, and T. Suemasu, CrystEngComm 16, 9590 (2014).

M.D. Levi and D. Aurbach, J. Electroanal. Chem. 421, 79 (1997).

P. Novák, F. Joho, R. Imhof, J.C. Panitz, and O. Haas, J. Power Sources 81-82, 212 (1999).

D.A. Stevens and J.R. Dahn, J. Electrochem. Soc. 147, 1271 (2000). 
42 A.C. Ferrari, Solid State Commun. 143, 47 (2007).

43 K. Zaghib, F. Brochu, A. Guerfi, and K. Kinoshita, J. Power Sources 103, 140 (2001).

44 Y.S. Hu, P. Adelhelm, B.M. Smarsly, S. Hore, M. Antonietti, and J. Maier, Adv. Funct. Mater. 17, 1873 (2007).

45 S.R. Sivakkumar, J.Y. Nerkar, and A.G. Pandolfo, Electrochim. Acta 55, 3330 (2010). 


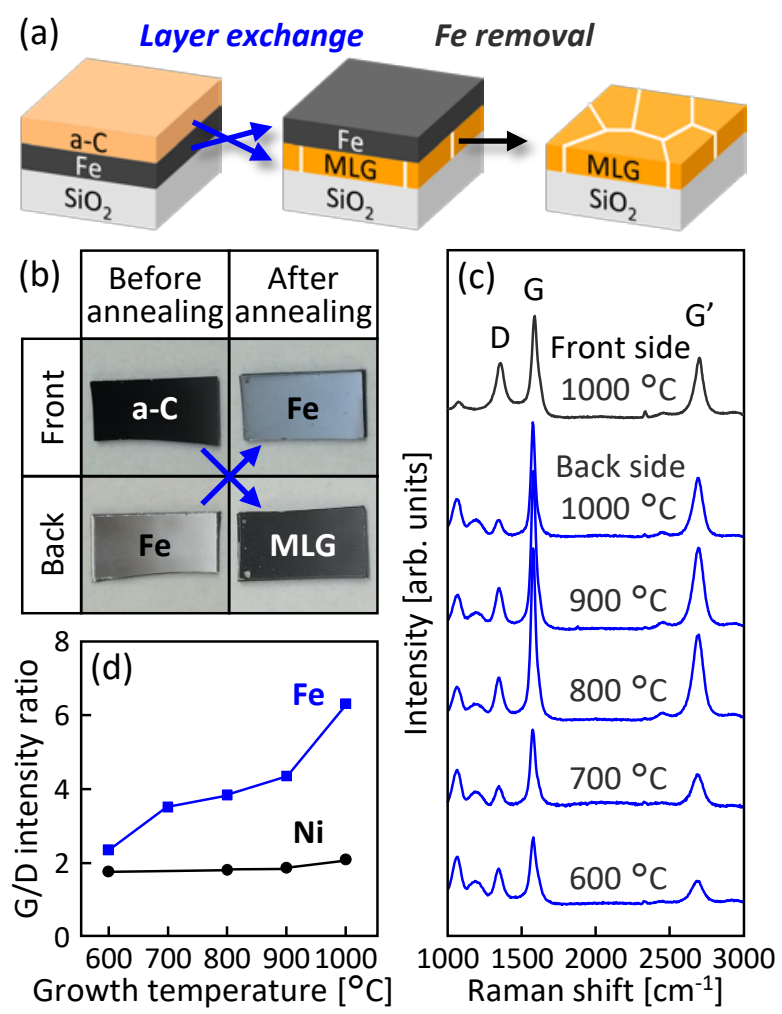

Fig. 1. Characteristics of the normal layer exchange sample. (a) Schematic of the sample preparation procedure. (b) Photographs of the front and back sides of the sample before and after annealing at $1000{ }^{\circ} \mathrm{C}$. (c) Raman spectra obtained from the front and back sides of the sample annealed at $600-1000{ }^{\circ} \mathrm{C}$. (d) Growth temperature dependence of the G/D intensity ratio of the Raman spectra, taken from the back sides of the samples. The data of the MLG formed by Ni-induced layer exchange are shown for comparison. ${ }^{30}$ 

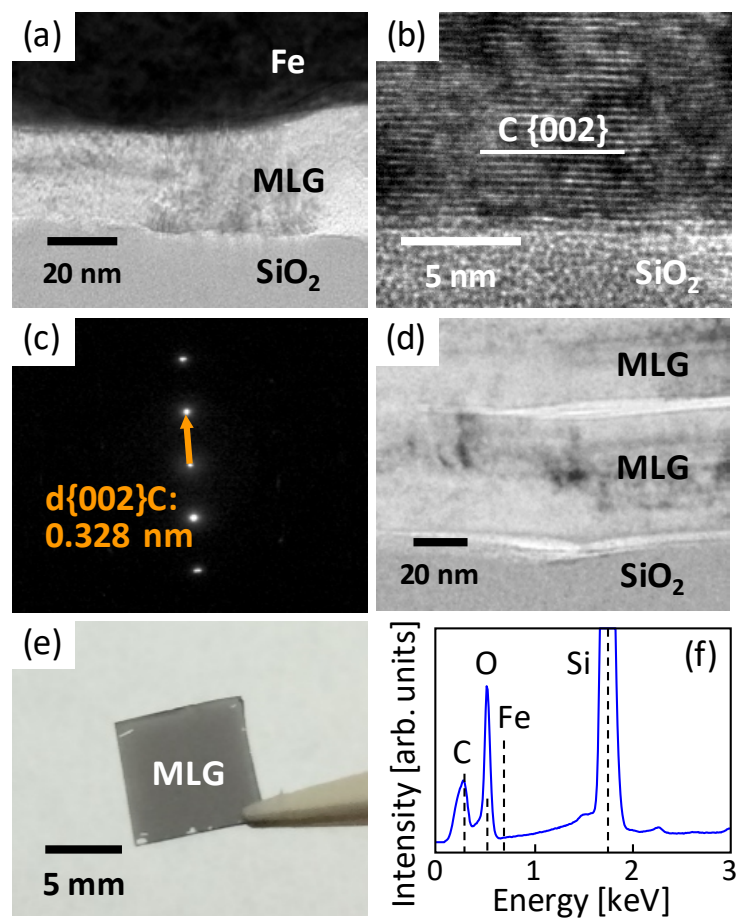

Fig. 2. Characteristics of the normal layer exchange sample formed at $1000{ }^{\circ} \mathrm{C}$. (a) Crosssectional bright-field TEM image. (b) High-resolution lattice image of the MLG layers showing a substrate-contacted region. (c) SAED pattern with a selected area of $200 \mathrm{~nm}$ diameter. (d) Bright-field TEM image showing an island area. (e) Photograph and (f) EDX spectrum of the sample after Fe removal. 
(a) Inverted layer exchange
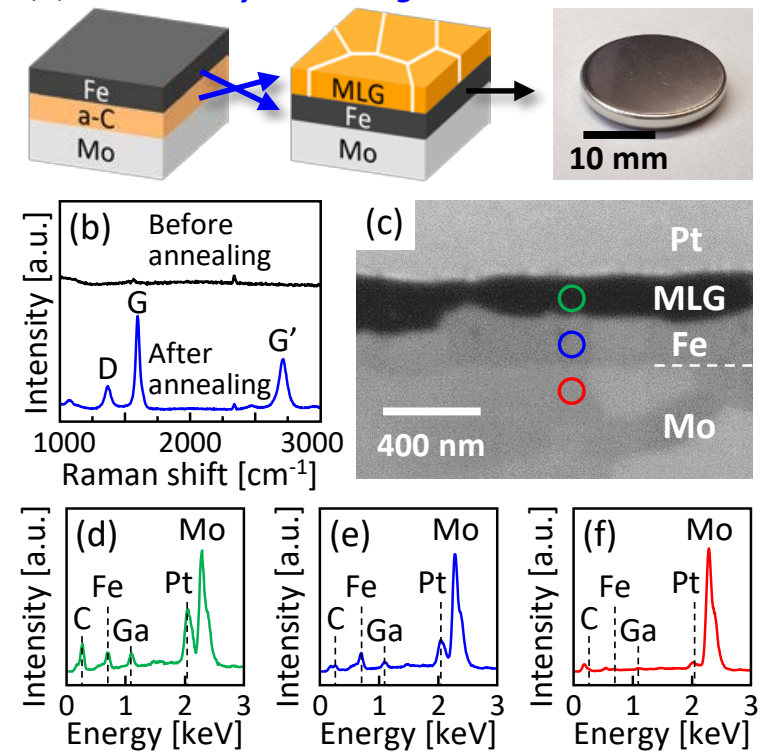

Fig. 3. Characteristics of the inverted layer exchange sample formed at $600{ }^{\circ} \mathrm{C}$. (a) Schematic of the sample preparation procedure and a photograph of a coin cell. (b) Raman spectra obtained from the front side of the sample before and after annealing. (c) Cross-sectional FIBSEM image, where the sample is tilted $52^{\circ}$. (d-f) EDX spectra of the sample, obtained at the circles in (c). Because the EDX measurement is performed on a tilted sample, the signals of the materials behind are also detected. 

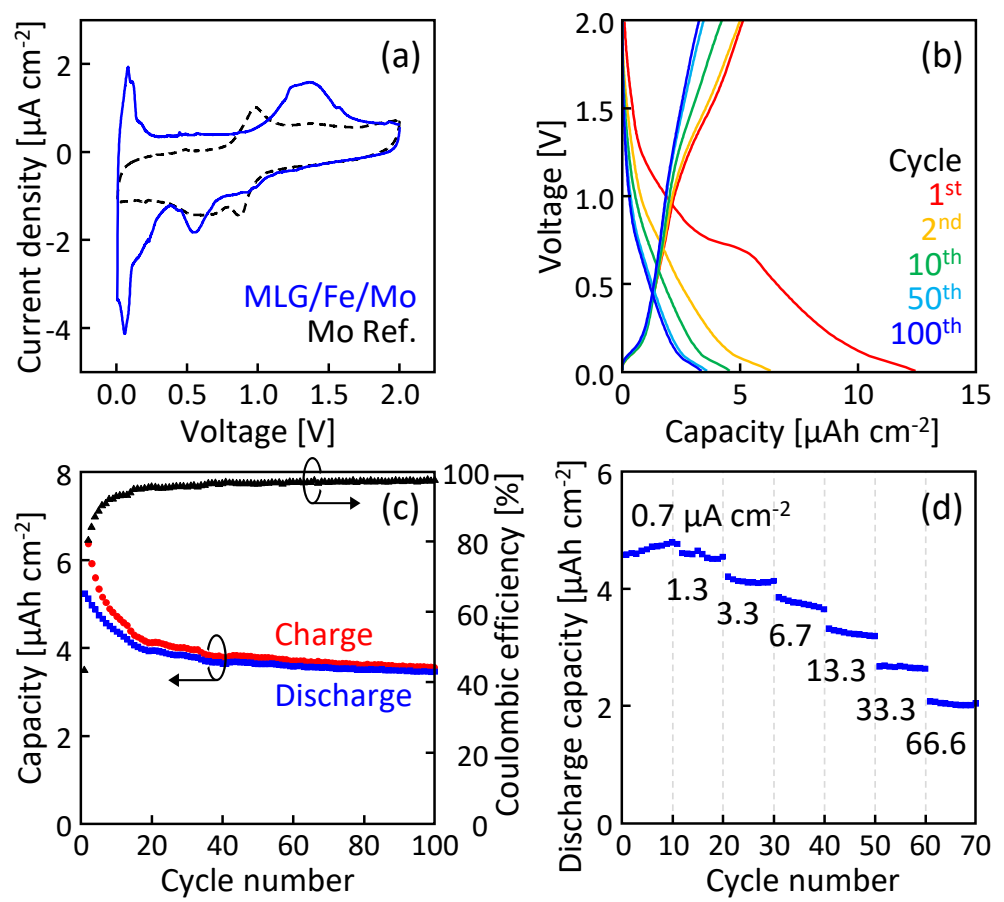

Fig. 4. Electrochemical characteristics of the coin cell using the MLG as an anode. (a) Cyclic voltammograms showing a second cycle at a scan rate of $50 \mu \mathrm{V} \mathrm{s}^{-1}$ in a voltage range of $0.005-$ 2.0 V. For comparison, the data for a bare Mo foil are also shown. (b) Galvanostatic charge/discharge cycles measured at a rate of $6.7 \mu \mathrm{A} \mathrm{cm}^{-2}$ in a voltage range of $0.005-2.0 \mathrm{~V}$. (c) Charge, discharge capacity, and coulombic efficiency as a function of cycle number. (d) Current-rate testing at various current densities $\left(0.7-66.6 \mu \mathrm{A} \mathrm{cm}^{-2}\right)$, every 10 cycles. 